\title{
New Medium-size Basis Sets for Rare-gas Atoms
}

\author{
Jan Makarewicz \\ Faculty of Chemistry, Adam Mickiewicz University \\ Grunwaldzka 6, 60-780 Poznań, Poland \\ e-mail: jama@amu.edu.pl
}

(Received: 10 February 2012; accepted: 6 June 2012; published online: 2 October 2012)

\begin{abstract}
New medium-size basis sets are constructed for rare-gas $(\mathrm{Rg})$ atoms and tested on their dimers $\mathrm{Rg}_{2}$. The main part of the these bases contains the standard aug-cc-pVTZ basis set modified and extended by the $g$ - and $h$-polarization functions. Their optimization allows accurate calculations of the dispersion interaction without the involvement of the bond functions. A new convenient analytical potential function is invented to fit accurately the interaction energy. The potentials derived for Rg dimers predict the vibrational transition energies and rotational constants to accuracy of $1 \%$.

Graphical abstract: New medium-size basis sets without the involvement of bond functions are constructed for raregas atoms and tested on their dimers.

Key words: $a b$ initio calculations, basis sets, interaction energy, optimized polarization functions, rare gas clusters, spectroscopic parameters
\end{abstract}

\section{INTRODUCTION}

Molecular clusters composed of a single molecule complexed by several rare-gas $(\mathrm{Rg})$ atoms have been a subject of intense experimental and theoretical investigations. Such clusters bound mainly by the dispersion interaction appeared to be most difficult to treat theoretically, since the dispersion energy arises solely from the interelectron correlation. Nevertheless, a good number of benchmark calculations employing sophisticated $a b$ initio methods have been reported for $\mathrm{Rg}$ dimers [1-15].

A major problem in these methods is the proper choice of the one-particle basis sets. A good description of dispersion needs extensive polarization and diffuse functions in these basis sets. Recently, very accurate interaction potentials for a series of $\mathrm{Rg}$ dimers have been calculated by employing large correlation consistent Dunning's basis sets aug-cc-pVXZ $[16,17]$ up to $X=5$ and 6 , supplemented with the diffuse bond functions located in the middle of the $\operatorname{Rg}_{2}$ bond $[12,14,15]$. However, the size of such extended basis sets makes the calculations on large $\mathrm{Rg}$ clusters unfeasible. An additional problem appears with the bond functions because it is not clear how they should be located in the space between the nuclei.
The goal of this work is to design moderate basis sets without the bond functions that could provide highly accurate results for the clusters containing $\mathrm{Rg}$ atoms. The lack of the bond functions can be compensated by the interaction-optimized polarization functions. To test the efficiency of the new basis sets, the interaction potentials for the $\mathrm{Rg}$ dimers will be calculated and expressed by a new convenient analytical form.

\section{METHOD}

The main source of errors in the calculated interaction energy is the incompleteness of the one-particle basis sets. Very large bases are necessary to achieve the complete basis set (CBS) limit. The basis size can be significantly reduced by optimizing the Gaussian parameters of the basis functions. The optimization is a difficult problem, so until now it has been performed only for $\mathrm{He}_{2}[2,6], \mathrm{Ne}_{2}$ [10] and HeNe [11] clusters.

A common practice is the optimization of only a small fraction of the basis functions from the total basis set. For example, Cybulski and coworkers $[12,15]$ constructed the basis sets for $\mathrm{Rg}$ dimers for $\mathrm{Rg}=\mathrm{He}, \mathrm{Ne} \mathrm{Ar}$ and $\mathrm{Kr}$ from the 
standard aug-cc-pVXZ bases (further abbreviated by avxz) supplemented by bond functions of the type proposed by Tao and Pan [18]. Only the bond functions located in the middle of the Rg-Rg bond were optimized in their bases.

We applied a similar method; however, we have not used the bond functions. To reduce significantly the basis size and simultaneously to conserve its flexibility, we have chosen the avtz basis as the main part. However, for $\mathrm{He}$ the larger avqz basis was selected because its structure is analogous to the avtz basis for heavier Rg atoms. These bases were modified by removing some tight polarization functions and by adding the $g$ - and $h$-functions. Our tests calculations for $\mathrm{He}_{2}$ and $\mathrm{Ne}_{2}$ indicated that the tight $d$-type functions with the highest exponent were insignificant in the bonding region, thus they were removed. However, they were conserved for $\mathrm{Ar}$ and $\mathrm{Kr}$. The $s$ - and $p$-type functions in the avtz and avqz bases were unchanged. As a consequence, the following structure of the basis sets was established: $s$ - and $p$-orbitals were taken from the original basis sets mentioned above, the three sets (four for $\mathrm{Kr}$ ) of $d$-, two sets of $f$-, one set of $g$ - and $h$-type functions were included into the desired basis sets. This basis structure will be abbreviated further by avqz(spd')211 for $\mathrm{He}$, $\operatorname{avtz(spd')211~for~Ne,~and~avtz(spd)211~for~Ar~and~Kr.~}$

\section{RESULTS AND DISCUSSION}

\section{1. Ab initio interaction energy}

The interaction energy of the $\mathrm{Rg}$ dimers has been calculated using the coupled cluster singles and doubles including the connected triples theory $[\operatorname{CCSD}(\mathrm{T})][19]$ in the framework of the supermolecular method, by employing the GAUSSIAN03 program package [20]. The basis set superposition error was eliminated by employing the usual counterpoise procedure [21]. As the core correlation has a small influence on the interaction energy, only the valence orbitals were involved in the correlation. Indeed, the core corrections at the equilibrium bond length $r_{\mathrm{e}}$, for $\mathrm{Ne}_{2}$ [13], $\mathrm{Ar}_{2}$ and $\mathrm{Kr}_{2}$ [14] were estimated to be about 0.2, 1 and $3 \mathrm{~cm}^{-1}$, respectively. The exponents of the polarization functions were optimized at the equilibrium bond length $r=r_{\mathrm{e}}$ because they depended weakly on $r$.

Table 1 presents the optimum exponents of the polarization functions. The optimum $d$-functions were established in different ways for different $\mathrm{Rg}$ atoms. For the $\mathrm{Ne}$ atom, the tightest set of $d$-functions was removed from the original avtz basis. Instead, one additional set of the diffuse $d$-functions with the exponent $\zeta=0.15$ was added. The two remaining $d$-sets were taken without a modification from the avtz basis. The $d$-functions for $\mathrm{He}$ were derived in an analogous way from the avqz basis. The three (four) $d$-sets for $\operatorname{Ar}(\mathrm{Kr})$ were left unchanged in the avtz basis. The tightest fourth contracted $d$-orbital of $\mathrm{Kr}$ is not presented in Table 1 for shortness. The $f$-functions obtained from the optimization appeared to be much more diffuse than those contained in the original basis sets. The same refers to the additional $g$ - and $h$-functions.

The obtained basis sets are not larger for Rg dimers, except for He, than the avtz +33221 basis sets with the bond functions $3 s 3 p 2 d 2 f 1 g[12,15]$. The size of the basis avtz +33221 quickly grows for the larger clusters $\mathrm{Rg}_{n}$ because the number of the bond functions located in the points placed between the nuclei grows as $n(n+1) / 2$. Our basis without the bond functions is more efficient. Nevertheless, it provides the accuracy comparable to that yielded by the larger avtz +33221 basis set.

Table 1. Orbital exponents $\zeta$ of the avqz(spd')211 and atz(spd')211 basis sets optimized for the Rg dimers

\begin{tabular}{c|l|l|l|l}
\hline$\zeta$ & $\mathrm{He}$ & $\mathrm{Ne}$ & $\mathrm{Ar}$ & $\mathrm{Kr}$ \\
\hline$d_{1}$ & 0.15 & 0.15 & $0.169^{\mathrm{a}}$ & $0.1257^{\mathrm{a}}$ \\
\hline$d_{2}$ & $0.351^{\mathrm{b}}$ & $0.386^{\mathrm{a}}$ & $0.410^{\mathrm{a}}$ & $0.3006^{\mathrm{a}}$ \\
\hline$d_{3}$ & $1.223^{\mathrm{b}}$ & $1.096^{\mathrm{a}}$ & $1.254^{\mathrm{a}}$ & $0.8084^{\mathrm{a}}$ \\
\hline$f_{1}$ & 0.25 & 0.25 & 0.20 & 0.15 \\
\hline$f_{2}$ & 0.75 & 0.75 & 0.60 & 0.45 \\
\hline$g, h$ & 0.30 & 0.40 & 0.25 & 0.20 \\
\hline
\end{tabular}

${ }^{\mathrm{a}} d$ - function from the standard avtz basis set

${ }^{\mathrm{b}} d$ - function from the standard avqz basis set

The interaction energy $E(r)$ calculated in this work in a wide range of the internuclear distance $r$ is compared in Tables 2-5 to the corresponding values calculated with the avtz +33221 and av5z +33221 bases $[12,15]$. The latter basis set provides the results close to the CBS limit within the frozen core approximation. Indeed, the errors $\Delta E(r)$ in the interaction energy calculated near the potential minimum by employing the basis av $5 z+33221$ are less than $0.1 \mu E_{h}$ for $\mathrm{He}_{2}$ and $\mathrm{Ne}_{2}$, and about $1 \mu E_{h}$ for $\mathrm{Ar}_{2}$ and $\mathrm{Kr}_{2}[12,14]$

Our results are less accurate in the repulsion region where $E(r)>0$. This is a consequence of the lack of the tight polarization functions in our bases. However, this drawback is not essential when the bound states of the clusters are considered, because the wave functions of such states are localized mainly in the bonding region where $E(r)<0$. 
Table 2. Comparison of the interaction energies $\left(\mu E_{h}\right)$ of $\mathrm{He}_{2}$ calculated at the bond length $r(\AA)$, using the avqz(spd')21, avtz +33221 and av $5 z+33221$ basis sets

\begin{tabular}{l|c|c|c}
\hline \multicolumn{1}{c|}{$r$} & $\begin{array}{c}\text { avqz(spd')21 } \\
110^{\mathrm{c}}\end{array}$ & $\begin{array}{c}\text { avtz }+33221^{\mathrm{b}} \\
91^{\mathrm{c}}\end{array}$ & $\begin{array}{c}\text { av5z }+33221^{\mathrm{b}} \\
205^{\mathrm{c}}\end{array}$ \\
\hline 1.75 & 5784.47 & 5769.68 & 5721.72 \\
\hline 2.000 & 1743.19 & 1734.72 & 1716.26 \\
\hline 2.250 & 454.30 & 449.81 & 443.24 \\
\hline 2.500 & 75.36 & 73.16 & 71.00 \\
\hline 2.750 & -19.37 & -20.38 & -21.08 \\
\hline 2.950 & -32.79 & -33.34 & -33.58 \\
\hline 2.975 & -32.97 & -33.48 & -33.68 \\
\hline 3.000 & -32.96 & -33.44 & -33.61 \\
\hline 3.025 & -32.79 & -33.23 & -33.37 \\
\hline 3.250 & -27.33 & -27.54 & -27.53 \\
\hline 3.50 & -19.55 & -19.60 & -19.57 \\
\hline 4.000 & -9.15 & -9.10 & -9.09 \\
\hline 5.000 & -2.28 & -2.27 & -2.26 \\
\hline
\end{tabular}

${ }^{\mathrm{a}}$ This work

${ }^{\mathrm{b}} \operatorname{Ref}[12]$

${ }^{\mathrm{c}}$ Dimension of the basis set

Table 3. Comparison of the interaction energies $\left(\mu E_{h}\right)$ of $\mathrm{Ne}_{2}$ calculated at the bond length $r(\AA)$, using the avtz(spd')211, avtz +33221 and av $5 z+33221$ basis sets

\begin{tabular}{|c|c|c|c|}
\hline$r$ & $\begin{array}{c}\operatorname{avtz}\left(\text { spd' }^{\prime}\right) 211^{\mathrm{a}} \\
118^{\mathrm{c}}\end{array}$ & $\begin{array}{c}\text { avtz }+33221^{b} \\
137^{\mathrm{c}}\end{array}$ & $\begin{array}{c}\text { av } 5 z+33221^{b} \\
299^{c}\end{array}$ \\
\hline 2.25 & 3670.73 & 3527.67 & 3444.36 \\
\hline 2.500 & 817.92 & 770.84 & 746.73 \\
\hline 2.750 & 42.74 & 26.76 & 23.99 \\
\hline 3.000 & -121.02 & -125.56 & -123.67 \\
\hline 3.075 & -129.68 & -132.05 & -129.96 \\
\hline 3.100 & -130.60 & -132.33 & -130.44 \\
\hline 3.125 & -130.77 & -131.92 & -130.13 \\
\hline 3.250 & -123.74 & -122.75 & -121.23 \\
\hline 3.500 & -94.18 & -92.29 & -90.91 \\
\hline 3.750 & -65.71 & -64.98 & -63.24 \\
\hline 4.000 & -44.74 & -44.66 & -42.83 \\
\hline 4.500 & -21.20 & -20.83 & -20.61 \\
\hline 5.000 & -10.80 & -10.47 & -10.54 \\
\hline
\end{tabular}

${ }^{\mathrm{a}}$ This work

${ }^{\mathrm{b}}$ Ref. [12]

${ }^{c}$ Dimension of the basis set

The errors calculated for $\mathrm{He}_{2}$ using our avqz(spd')21 basis are well below $1 \mu E_{h}$ in a large domain of the bonding region, for $r>r_{\mathrm{e}}$. The additional set of the $h$-functions reduces the error in this region to less than $0.3 \mu E_{h}$, so the $h$-functions are excluded from the presented basis set.
Table 4. Comparison of the interaction energies $\left(\mu E_{h}\right)$ of $\mathrm{Ar}_{2}$ calculated at the bond length $r(\AA)$, using the avtz(spd)211, avtz +33221 and av5z +33221 basis sets

\begin{tabular}{c|c|c|c}
\hline$r$ & $\begin{array}{c}\text { avtz (spd)211 } \\
140^{\mathrm{c}}\end{array}$ & $\begin{array}{c}\text { avtz }+33221^{\mathrm{b}} \\
145^{\mathrm{c}}\end{array}$ & $\begin{array}{c}\text { av5z }+33221^{\mathrm{b}} \\
307^{\mathrm{c}}\end{array}$ \\
\hline 3.000 & 3330.30 & 3175.30 & 3054.98 \\
\hline 3.250 & 628.64 & 557.65 & 518.11 \\
\hline 3.500 & -239.38 & -276.99 & -278.98 \\
\hline 3.750 & -429.88 & -451.80 & -440.82 \\
\hline 3.775 & -432.56 & -453.26 & -441.87 \\
\hline 3.800 & -433.42 & -453.03 & -441.32 \\
\hline 3.850 & -430.76 & -448.30 & -436.21 \\
\hline 4.000 & -398.84 & -411.42 & -399.60 \\
\hline 4.250 & -314.76 & -322.75 & -313.12 \\
\hline 4.500 & -233.62 & -239.45 & -232.30 \\
\hline 5.000 & -122.88 & -123.19 & -122.96 \\
\hline 6.000 & -37.66 & -37.81 & -37.77 \\
\hline 7.000 & -14.02 & -14.01 & -14.03 \\
\hline
\end{tabular}

${ }^{\mathrm{a}}$ This work

${ }^{b}$ Ref. [12]

${ }^{\mathrm{c}}$ Dimension of the basis set

Table 5. Comparison of the interaction energies $\left(\mu E_{h}\right)$ of $\mathrm{Kr}_{2}$ calculated at the bond length $r(\AA)$, using the avtz(spd)211, avtz +33221 and av $5 z+33221$ basis sets

\begin{tabular}{|c|c|c|c|}
\hline$r$ & $\begin{array}{c}\operatorname{avtz}(\operatorname{spd}) 211^{\mathrm{a}} \\
158^{\mathrm{c}}\end{array}$ & $\begin{array}{c}\text { avtz }+33221^{b} \\
163^{\mathrm{c}}\end{array}$ & $\begin{array}{c}\operatorname{av} 5 z+33221^{b} \\
325^{c}\end{array}$ \\
\hline 3.250 & 3866.9 & 3691.62 & 3579.74 \\
\hline 3.500 & 764.0 & 671.39 & 643.47 \\
\hline 3.750 & -317.6 & -369.74 & -361.52 \\
\hline 4.000 & -588.1 & -617.24 & -599.07 \\
\hline 4.025 & -593.9 & -621.50 & -603.08 \\
\hline 4.050 & -597.6 & -623.46 & -604.86 \\
\hline 4.075 & -598.8 & -623.38 & -604.69 \\
\hline 4.250 & -566.8 & -583.32 & -565.19 \\
\hline 4.500 & -463.7 & -473.40 & -458.24 \\
\hline 4.750 & -355.3 & -360.56 & -349.83 \\
\hline 5.000 & -265.1 & -267.23 & -260.72 \\
\hline 6.000 & -82.2 & -81.82 & -81.21 \\
\hline 8.000 & -12.8 & -12.48 & -12.62 \\
\hline
\end{tabular}

${ }^{\mathrm{a}}$ This work

${ }^{\mathrm{b}}$ Ref. [15]

${ }^{\mathrm{c}}$ Dimension of the basis set

For $\mathrm{Ne}_{2}$, our basis avtz(spd')211, slightly smaller than the avtz +33221 basis [12] yields more accurate $E(r)$ values near the potential minimum. Both basis sets show a similar behavior at large internuclear distances. 
Table 6. Potential parameters determined for the Rg dimers, using our (I) and av5z+33221 (II) basis sets

\begin{tabular}{l|c|c|c|c|c|c|c|c}
\hline & \multicolumn{2}{|c|}{$\mathrm{He}_{2}$} & \multicolumn{2}{c|}{$\mathrm{Ne}_{2}$} & \multicolumn{2}{c}{$\mathrm{Ar}_{2}$} & \multicolumn{2}{c}{$\mathrm{Kr}_{2}$} \\
\hline \multicolumn{1}{c|}{ Parameter } & $\mathrm{I}$ & $\mathrm{II}^{\mathrm{a}}$ & $\mathrm{I}$ & $\mathrm{II}^{\mathrm{a}}$ & $\mathrm{I}$ & $\mathrm{II}^{\mathrm{a}}$ & $\mathrm{I}$ & $\mathrm{II}^{\mathrm{b}}$ \\
\hline$r_{\mathrm{e}}(\AA)$ & 2.9852 & 2.9755 & 3.1167 & 3.0993 & 3.8009 & 3.7784 & 4.0808 & 4.0601 \\
\hline$r_{\mathrm{e}}(\AA)^{\mathrm{c}}$ & & 2.9764 & & 3.0988 & & 3.7785 & & 4.0592 \\
\hline$a_{0}$ & 6.2046 & 6.2081 & 6.3641 & 6.3864 & 6.4062 & 6.3757 & 6.3878 & 6.3799 \\
\hline$a_{1}$ & -1.2585 & -1.2234 & -1.4472 & -1.4557 & -0.8901 & -0.8508 & -0.7302 & -0.7241 \\
\hline$a_{2}$ & -1.7753 & -1.7476 & -1.1845 & -1.3464 & -1.7838 & -1.4465 & -1.6771 & -1.6091 \\
\hline$\beta$ & $1.0000^{\mathrm{d}}$ & $1.0000^{\mathrm{d}}$ & $1.0000^{\mathrm{d}}$ & $1.0000^{\mathrm{d}}$ & 1.0530 & 1.2046 & 1.1508 & 1.1956 \\
\hline$D_{\mathrm{e}}\left(\mu E_{\mathrm{h}}\right)$ & 33.01 & 33.70 & 130.89 & 130.41 & 433.40 & 441.88 & 598.98 & 605.02 \\
\hline$D_{\mathrm{e}}\left(\mu E_{\mathrm{h}}\right)^{\mathrm{c}}$ & & 33.67 & & 130.33 & & 441.90 & & 604.89 \\
\hline
\end{tabular}

${ }^{\mathrm{c}}$ Parameter determined for $\mathrm{He}_{2}, \mathrm{Ne}_{2}, \mathrm{Ar}_{2}$ in Ref. [12], and for $\mathrm{Kr}_{2}$ in Ref. [15]

${ }^{\mathrm{d}}$ Parameter constrained

The errors $\Delta E(r)$ produced by avtz(spd)211 and avtz +33221 basis sets for $\mathrm{Ar}_{2}$ are comparable in magnitude $\left(\approx 10 \mu E_{h}\right)$ near the equilibrium, but their signs are the opposite. For $r>r_{e}$ the $\operatorname{avtz}(\mathrm{spd}) 211$ basis provides more accurate results. A similar situation takes place for $\mathrm{Kr}_{2}$, however $\Delta E(r)$ calculated for avtz +33221 basis sets are about twice larger than for $\mathrm{Ar}_{2}$.

\section{2. Analytical potential energy curves}

To derive the measurable molecular parameters from the single-point energy values they should be converted to the analytical potential energy curve $V(r)$. Usually, $V(r)$ is composed of a short-range repulsive $V_{+}$and a long-range attractive $V_{-}$parts. The attractive potential can be represented by the damped dispersion series containing the dispersion coefficients $C_{2 n}$ [22]. The repulsive potential can be well approximated, according to the Born-Mayer model, by the exponential function $V_{+}=A \exp (-b r)$ or by its modified form $\tilde{V}_{+}=A \exp \left(-b r+c r^{2}\right)$ [5]. From the potential parameters $A, b, c$ and $C_{2 n}$, one can determine the binding energy $D_{e}$ and the equilibrium bond length $r_{\mathrm{e}}$ that characterize the potential $V(r)$. However, as noticed by Tang and Toennies [22], the potential parameters $A$ and $b$ are extremely sensitive to $D_{e}$ and $r_{e}$.

Thus, it would be desirable to find the analytical form of $V(r)$ parametrized directly by $D_{e}$ and $r_{e}$. The commonly known Morse potential

$$
V_{M}(r)=D_{e}\left[y^{2}(r)-1\right]
$$

where $y(r)=1-\exp (-a x)$ and $x=\left(r-r_{e}\right) / r_{e}$ fulfils this condition; however, it is not accurate in a long-range domain. This deficiency can be repaired by including the damped dispersion terms into (1) or by modifying the original potential $V_{M}(r)$ in a different way.

We have found that $V_{M}(r)$ can be significantly improved by treating the parameter $a$ in (1) as a function of $r$ in the following form

$$
a(r)=a_{0}+a_{1} \tanh (\beta x)+a_{2} \tanh ^{2}(\beta x)
$$

with the adjustable parameter $\beta$. Such a modified Morse potential conserves the advantages of the original one and simultaneously allows an accurate fit of the $a b$ initio energy values in a large domain of $r$ to a high accuracy, even without additional dispersion terms. Moreover, we have found that the function $a(r)$ in the form (2) remains to work properly also for covalently bound molecules.

The potential parameters $D_{e}, r_{e}$, and $a_{k}$ fitted to the energy values given in Tables 2-5 are collected in Table 6 . The energy values calculated using the av $5 z+33221$ basis set $[12,15]$ were fitted with the standard errors of about $0.05 \mu E_{h}$ for all the considered dimers. The differences in the binding energies $D_{e}$ determined in Refs. $[12,15]$ and in this work are also of the same order of magnitude. Only for $\mathrm{Kr}_{2}$, this difference is slightly larger $\left(0.13 \mu E_{h}\right)$. The differences in $r_{e}$ are less than $0.001 \AA$. These test results demonstrate that the modified Morse potential is competitive to the standard $V_{+}+V_{-}$representation.

The potential functions derived for our basis sets are less accurate. The potential minima are shifted by about $0.02 \AA\left(0.01 \AA\right.$ for $\left.\mathrm{He}_{2}\right)$ towards larger distances, and the binding energies $D_{e}$ calculated using our basis sets deviate from the corresponding $D_{e}(\operatorname{av} 5 \mathrm{z}+33221)$ values by about $(-0.1,+0.1,-1.9,-1.3) \mathrm{cm}^{-1}$ for $\mathrm{He}, \mathrm{Ne}, \mathrm{Ar}$, and $\mathrm{Kr}$, respectively. Such errors are comparable to the core corrections, usually neglected in the calculations of the interaction energies. 
Table 7. Vibrational transition frequencies $\left(\mathrm{cm}^{-1}\right)$ and rotational constants $B_{0}\left(\mathrm{~cm}^{-1}\right)$ calculated for the Rg dimers, using our (I) and av $5 z+33221$ (II) basis sets

\begin{tabular}{c|c|c|c}
\hline Dimer & $v^{\prime}-v^{\prime \prime}$ & $\mathrm{I}^{\mathrm{a}}$ & $\mathrm{II}^{\mathrm{b}}$ \\
\hline $\mathrm{Ne}_{2}$ & $1-0$ & 13.56 & 13.46 \\
\hline & $B_{0}$ & 0.1532 & 0.1547 \\
\hline $\mathrm{Ar}_{2}$ & $1-0$ & 24.99 & 25.27 \\
\hline & $2-1$ & 19.87 & 20.11 \\
\hline & $3-2$ & 14.92 & 15.15 \\
\hline $\mathrm{Kr}_{2}$ & $B_{0}$ & 0.05641 & 0.05708 \\
\hline & $1-0$ & 20.61 & 20.78 \\
\hline & $2-1$ & 18.48 & 18.60 \\
\hline & $3-2$ & 16.37 & 16.45 \\
\hline & $4-3$ & 14.29 & 14.33 \\
\hline & $5-4$ & 12.24 & 12.27 \\
\hline & $B_{0}$ & 0.02369 & 0.02390 \\
\hline
\end{tabular}

\section{${ }^{\mathrm{a}}$ This work}

${ }^{\mathrm{b}}$ Calculated for $\mathrm{Ne}_{2}$ and $\mathrm{Ar}_{2}$ in Ref. [12], and for $\mathrm{Kr}_{2}$ in Ref. [15]

The quality of the potentials presented was tested from the spectroscopic point of view. Table 7 collects the frequencies of the vibrational transitions as well as the ground-state rotational constants $B_{0}$ of the dimers $\mathrm{Rg}_{2}$, except for $\mathrm{He}_{2}$ for which no bound states were found. These quantities were calculated by solving numerically the Schrödinger equation for the nuclear motion. A comparison of the results derived for the avtz(spd)211 and av $5 z+33221$ basis sets shows that the vibrational frequencies obtained for our basis set deviate by about $1 \%$ or less from more accurate values reported $[12,15]$ for the latter basis set. The same refers for the rotational constants. This shows that our basis sets allow us to generate the potentials useful for spectroscopic purposes.

\section{Acknowledgments}

Part of the calculations has been performed at Poznan Supercomputing and Networking Center.

\section{References}

[1] D.E. Woon, J. Chem. Phys. 100, 2838 (1994).

[2] T. van Mourik, J.H. van Lenthe, J. Chem. Phys. 102, 7479 (1995).

[3] W. Klopper, J. Noga, J. Chem. Phys. 103, 6127 (1995).

[4] J.V. Burda, R. Zahradnik, P. Hobza, M. Urban, Mol. Phys. $89,425(1996)$

[5] T. Korona, H.L. Williams, R. Bukowski, B. Jeziorski, K. Szalewicz, J. Chem. Physics, 106, 5109 (1997).

[6] J. Komasa, J. Rychlewski, Mol. Phys. 91, 909 (1997).

[7] B. Fernández, H. Koch, J. Chem. Phys. 109, 10255 (1998).

[8] T. van Mourik, A.K. Wilson, H. Dunning, Jr., Mol. Phys. 96, 529 (1999).

[9] J. Komasa, J. Chem. Phys. 110, 7909 (1999).

[10] J. van de Bovenkamp, F.B. van Duijneveldt, J. Chem. Phys. 110, 11141 (1999).

[11] J. van de Bovenkamp, T. van Mourik, F.B. van Duijneveldt, Mol. Phys. 487, 97 (1999).

[12] S.M. Cybulski, R.R. Toczyłowski, J. Chem. Phys. 111, 10520 (1999).

[13] R.J. Gdanitz, Chem. Phys. Lett. 348, 67 (2001).

[14] P. Slaviček, R. Kalus, P. Paška, I. Odvárková, P. Hobza, A. Malijevský, J. Chem. Phys. 119, 2102 (2003).

[15] T.P. Hale, S.M. Cybulski, J. Chem. Phys. 119, 5487 (2003).

[16] T.H. Dunning, Jr., J. Chem. Phys. 90, 1007 (1989).

[17] D.E. Woon, T.H. Dunning, Jr., J. Chem. Phys. 100, 2975 (1994).

[18] F.-M. Tao, Y.-K. Pan, Chem. Phys. Lett. 194, 162 (1992).

[19] K. Raghavachari, G.W. Trucks, J.A. Pople, M. Head-Gordon, Chem. Phys. Lett. 157, 479 (1989).

[20] M.J. Frish et al., GAUSSIAN-03 Inc., Pittsburgh PA, 2003.

[21] S.F. Boys, F. Bernardi, Mol. Phys. 19, 553 (1970).

[22] K.T. Tang, J.P. Toennies, J. Chem. Phys. 118, 4976 (2003).

JAN MAKarewiCZ works at the Faculty of Chemistry of Adam Mickiewicz University in Poznań. There, he received his MSc degree, $\mathrm{PhD}$ and then DSc in Physical and Theoretical Chemistry. He has been collaborating with theoretical and experimental groups from Italy, France, Russia, Spain, and Switzerland. He develops theoretical and computational methods of a high resolution rovibrational molecular spectroscopy. 